\title{
Ashtekar Variables: Structures in Bundles
}

\author{
Christian Fleischhack ${ }^{1,2, *}$ and Philipp Levermann ${ }^{2,3, *}$ \\ ${ }^{1}$ Institut für Mathematik \\ Universität Paderborn \\ Warburger Straße 100 \\ 33098 Paderborn, Germany \\ ${ }^{2}$ Department Mathematik \\ Universität Hamburg \\ Bundesstraße 55 \\ 20146 Hamburg, Germany \\ ${ }^{3}$ II. Institut für Theoretische Physik \\ Universität Hamburg \\ Luruper Chaussee 149 \\ 22761 Hamburg, Germany
}

December 6, 2011

\begin{abstract}
Canonical gravity can be formulated by means of a densitized dreibein together with an $\mathrm{SU}(2)$ connection. These so-called Ashtekar variables are the fundamental quantities, loop quantum gravity is resting on. In this paper we review these variables from the perspective of fibre bundles. This is straightforward for the dreibein field as this is simply a frame. The Ashtekar connection, however, is more complicated. It turns out, that at the level of the tangent bundle, it is given by the Levi-Civita connection plus a multiple of the Weingarten mapping, whose action on vector fields is induced from the vector product on $\mathbb{R}^{3}$. Lifted to the spin bundle, one regains the well-known $\mathrm{SU}(2)$ Ashtekar connection. At the end, we apply our results to FRW spacetimes.
\end{abstract}

\section{Introduction}

25 years ago, Abhay Ashtekar [2, 1] introduced new variables to describe canonical gravity. Their main advantage has been that they drastically simplified the constraints of gravity: These become polynomial. This opened a completely new door to approach the quantization of gravity, ultimately leading to loop quantum gravity. Over the last quarter of a century, there has been only a single, but significant extension of Ashtekar's variables. In the mid90s, Barbero [3] and Immirzi [9] added a new parameter $\beta$, with $\beta=\mathrm{i}$ giving the original variables. It turned now out a big advantage that for real $\beta$ the structure group is no longer $\mathrm{Sl}_{\mathbb{C}}(2)$, but $\mathrm{SU}(2)$. This has been crucial for the integration theory of loop quantum gravity.

*e-mail: fleischh@math.upb.de, karl.philipp.levermann@desy.de 
Before the Barbero-Immirzi idea has been introduced, complicated reality condition had been necessary to implement the real structure of the theory. However, the new formulation has the drawback that the Hamiltonian constraint is no longer polynomial. This has only been cured by the Thiemann trick [15], rewriting the term with prefactor $1+\beta^{2}$ by means of certain Poisson brackets. That combined the advantages of integration theory on compact structure groups and the functional analysis of polynomial constraints.

Despite the fundamental rôle of Ashtekar's variables, their geometric origin have remained open. To the best of our knowledge, only local versions using sophisticated index notations have been available so far. The present paper aims at a first glimpse of the differential geometry underlying Ashtekar's variables. These are a connection in some principal fibre bundle to be determined and a densitized dreibein field. The latter one is rather easy to state, whence we will focus on the connection variables which form the configuration space of the theory (up to gauge transformations). More precisely, we will describe the principal fibre bundle the connection lives in, and then discuss Ashtekar-type connections. Additionally, we present a very simple formulation of these variables in the context of Friedmann-RobertsonWalker models. In a forthcoming paper we will focus on the description of constraints.

The present paper is organized as follows: We start in Section 2 with a short review of canonical gravity mostly to fix the notations in Section 3, and comment, in Section 4, briefly on densitized frame fields being one part of Ashtekar's variables. The rest of this paper is devoted to the Ashtekar connection. Sloppily, on tangent space level, this connection is the sum of the Levi-Civita connection and the Weingarten mapping (augmented by the Barbero-Immirzi parameter), the latter one to be described in Subsection [5.2. However, to make this construction precise, we need to transfer the $\mathbb{R}^{3}$ vector product to a certain product on the tangent space (of a 3D manifold) to be given in Subsection 5.1. This leads to the Ashtekar $\mathrm{SO}(3)$ connection in Subsection 5.6. Introducing spin structures in Section 6 , we get the usual Ashtekar SU(2) connection. Finally, we will apply the whole construction to homogeneous isotropic spacetimes in Section 7 .

\section{Canonical Gravity}

Classically, gravity [16, 14] is described within Einstein's theory of general relativity by a smooth metric $g$ of Lorentzian signature on a 4-dimensional manifold satisfying Einstein's equations

$$
R_{\mu \nu}-\frac{1}{2} g_{\mu \nu} R+g_{\mu \nu} \Lambda=\frac{8 \pi \gamma}{c^{4}} T_{\mu \nu}
$$

Here, $R_{\mu \nu}$ is the Ricci tensor w.r.t. $g$, and $R$ the corresponding Ricci scalar. $\Lambda$ denotes the cosmological constant. The right-hand side describes the matter content of the universe; we will assume this term to vanish throughout our paper (vacuum case). We neglect $\Lambda$ as well. In this paper we will restrict ourselves to a particular class of such manifolds - that of so-called spacetimes.

Definition 2.1 A spacetime is a 4-dimensional, connected, time-oriented and oriented Lorentz manifold.

Often, physical theories are quantized in their canonical form as this admits an initial-value formulation. A key feature of classical general relativity is, however, the unified treatment of space and time - or, rephrased, the lack of a standard splitting into space and time. Thus, instead of having a "canonical" initial hypersurface at $t=0$, any spacelike hypersurface might serve as initial hypersurface.

For this, let us assume we are given some Lorentzian metric $g$ on $M$ fulfilling the vacuum Einstein equations, and some spacelike hypersurface $\Sigma$ embedded into $M$. The geometry of 
$\Sigma$ is determined by the Riemannian metric $q$ induced by $g$ and by the second fundamental form $K$. The latter one is a symmetric bilinear form on $\Sigma$ and describes the shaping of $\Sigma$ within the spacetime $M$; it is also called "extrinsic curvature" in physics. However, $q$ and $K$ cannot be any metric and any form. In fact, they are induced from a metric on $M$ fulfilling the Einstein equations. Therefore, the Gauß-Codazzi equations impose constraints on $(q, K)$.

It has been a big issue whether these constraints are not only necessary, but also sufficient for a Cauchy evolution. In her celebrated paper [6], Choquet-Bruhat showed that indeed the Cauchy problem of general relativity has locally a unique solution. The constraints are sufficient to locally reconstruct the spacetime uniquely up to isometric isomorphisms from a Riemannian metric $q$ and a symmetric bilinear form $K$ on some 3-manifold $\Sigma$. [14, 13, Only globally, the situation is a bit more subtle. Usually, these problems can be circumvented by some additional physical assumptions. To avoid, in particular, multiple intersections with timelike curves, one has restricted oneself to a special type of spacetimes - so-called globally hyperbolic ones. This means, by definition [5], that $M$ fulfills the strong causality condition and for all $p, q \in M$ the intersection of the causal future of $p$ and the causal past of $q$ is compact. Indeed, Geroch [8] has shown that any globally hyperbolic spacetime has a Cauchy slice, i.e., some spacelike hypersurface of $M$ that is intersected exactly once by each inextendible timelike curve in $M$ [5]; moreover then $M \cong \mathbb{R} \times \Sigma$. However, Geroch was only able to prove this in the $C^{0}$ category. It is still a rather recent result, that this statement is also true in the smooth category.

Theorem 2.1 If a spacetime $(M, g)$ is globally hyperbolic, then it is isometric to

$$
\left(\mathbb{R} \times \Sigma,-f \mathrm{~d} \tau^{2}+g_{\tau}\right)
$$

with a smooth positive function $f: \mathbb{R} \longrightarrow \mathbb{R}$ and a smooth family of Riemannian metrics $g_{\tau}$ on $\Sigma$. Moreover, each $\{t\} \times \Sigma$ is a Cauchy slice. [5, 4]

\section{Notation}

Unless specified otherwise, let $\Sigma$ be some oriented three-dimensional manifold, and let $q$ be some Riemannian metric $q$ on $\Sigma$. We write $\langle X, Y\rangle:=q(X, Y)$ for all vector fields $X, Y$ on $\Sigma$. As $\Sigma$ is orientable, it has a spin structure and is parallelizable. [10] Next, we assume that $M$ is some manifold diffeomorphic to $\Sigma \times \mathbb{R}$, whereas we identify $\Sigma$ with $\Sigma \times\{0\}$. Moreover, $g$ is a metric on $M$ inducing $q$ on $\Sigma$. We will denote $g(X, Y)$ again by $\langle X, Y\rangle$; this does not apply to Section 7. Finally, observe that we are always working with smooth objects only.

\section{Frames}

One part of the Ashtekar variables is formed by a densitized dreibein, where dreibeine are orthonormal frames on a three-dimensional manifold $\Sigma$. In contrast to the Ashtekar connections to be defined later, the definition of frames works in any dimension $n$.

\subsection{General Frames}

Definition 4.1 A frame at $x \in \Sigma$ is a vector space isomorphism

$$
e: \mathbb{R}^{n} \longrightarrow T_{x} \Sigma \text {. }
$$

Another way to specify a frame is to select a basis of $T_{x} \Sigma$. 11] Recall that the frame bundle on $\Sigma$ is given by the disjoint union, indexed by $x \in \Sigma$, of all frames at $x$. The differentiable 
structure on it is naturally induced from that on $\Sigma$ by decomposing each frame w.r.t. some appropriate local coordinate system on $T \Sigma$. The resulting bundle $\operatorname{Gl}(\Sigma)$ is independent of such a choice and called frame bundle. Its structure group is given by $\operatorname{Gl}(n)$. Frames are simply (local) sections in the frame bundle.

If we choose some (local) basis for $T_{x} \Sigma$ (and the canonical basis of $\mathbb{R}^{n}$ ), then any frame $e$ at $x$ as being a vector space isomorphism, is characterized by some matrix. Its determinant will be called determinant det $e$ of $e$. If we choose a different basis on $T_{x} \Sigma$, we might get an additional factor. In fact, the transformation matrix intertwining two bases is some $\operatorname{Gl}(n)$ element, whose determinant is precisely that factor. Note that this prefactor may change from point to point, if we consider general local frames. In the case we are interested in, however, the tangent bundle will be globally trivial, such that we may assume that each frame is globally defined and such a change of bases corresponds to multiplication by some function on full $\Sigma$.

To finally arrive at the definition of Ashtekar fields, observe that multiplication of any tensorial object with $(\operatorname{det} e)^{-k}$ gives the corresponding tensor density of weight $k$.

Definition 4.2 The Ashtekar field $E$ to a frame $e$ is the densitized frame field

of weight 1.

$$
E:=\frac{1}{\operatorname{det} e} e
$$

Observe that this definition depends on the choice of a basis on each $T_{x} \Sigma$. If that basis is given by the image of the canonical basis on $\mathbb{R}^{n}$, then $\operatorname{det} e$ is, of course, 1 . If $n \neq 1$, then the frame can be reconstructed from the Ashtekar field. Use

to obtain

$$
\operatorname{det} E=\operatorname{det}\left(\frac{1}{\operatorname{det} e} e\right)=\left(\frac{1}{\operatorname{det} e}\right)^{n} \operatorname{det} e=(\operatorname{det} e)^{1-n}
$$

$$
e=(\operatorname{det} e) E=(\operatorname{det} E)^{\frac{1}{1-n}} E
$$

\subsection{Orthonormal Frames}

Definition 4.3 A frame is called

- orthonormal w.r.t. the metric $q$ on $\Sigma$ iff it is an isometry 1 ;

- oriented iff it preserves the orientation.

Again, one may specify an orthonormal frame by an orthonormal basis of $T_{x} \Sigma$. [11]

Any frame defines a metric such that the frame is orthonormal w.r.t. that metric. In fact, if $\langle\cdot, \cdot\rangle_{\text {Eucl }}$ denotes the Euclidean scalar product, then

$$
q(X, Y):=\left\langle e^{-1}(X), e^{-1}(Y)\right\rangle_{\text {Eucl }} \quad \text { for } X, Y \in T_{x} \Sigma
$$

defines a metric $q$ on $\Sigma$, such that $e$ is an isometry. While frames determine a metric uniquely, a metric does not fix the orthonormal frame. In fact, $e$ and $e^{\prime}$ are isometries for $q$ iff $e^{\prime}=e \circ L_{g}$ for some $g \in \mathrm{O}(n)$, where $L_{g}$ denotes the left translation by $g$.

The bundle $\mathrm{O}_{q}(\Sigma)$ of orthonormal frames can be defined completely analogously to that of general frames; one only has to replace $\mathrm{Gl}(n)$ by $\mathrm{O}(n)$. At the same time, $\mathrm{O}_{q}(\Sigma)$ is the reduction of the structure group $\mathrm{Gl}(n)$ of the frame bundle to the structure group $\mathrm{O}(n)$. Similar arguments apply to the bundle $\mathrm{O}_{q}^{+}(\Sigma)$ of oriented orthonormal frames having structure group $\mathrm{SO}(n)$.

\footnotetext{
${ }^{1}$ Here, as throughout the paper, we have equipped $\mathbb{R}^{n}$ with the standard Euclidean metric.
} 


\section{Ashtekar Connection}

The other part of the Ashtekar variables is a metric connection on the tangent bundle - or in an appropriate associated principal fibre bundle. In each case, we continue to be given some Riemannian metric $q$ on some oriented manifold $\Sigma$. As we need a vector-product like structure on $T \Sigma$, the dimension of $\Sigma$ is now required to be 3 . Later, for the definition of the Weingarten mapping, we assume $\Sigma$ to be an embedded submanifold of a four-dimensional Lorentzian manifold $(M, g)$, whereas the metric $q$ on $\Sigma$ is induced by $g$ to $\Sigma$.

\subsection{Induced Vector Product}

First we transfer the vector product on $\mathbb{R}^{3}$ by means of the metric to $T \Sigma$.

Definition 5.1 Let $q$ be a metric on $\Sigma$, and let $X, Y$ be tangent vectors on $\Sigma$. For any oriented $q$-orthonormal frame $e$ define

$$
X \bullet Y:=e\left[e^{-1}(X) \times e^{-1}(Y)\right],
$$

with $\times$ being the standard vector product on $\mathbb{R}^{3}$.

Lemma 5.1 • is well defined as it depends on $q$ only, but not on the oriented $q$-orthonormal frame $e$ itself.

Proof If $\varepsilon, \eta$ are inverses of oriented $q$-orthonormal frames, then there is an $A \in \mathrm{SO}(3)$ with $\eta(X)=A \varepsilon(X)$ for all $X$. Hence, by the $\mathrm{SO}(3)$ invariance of the vector product, we have

$$
\begin{aligned}
\eta\left[X \bullet_{\varepsilon} Y\right] & =A \varepsilon\left[X \bullet_{\varepsilon} Y\right]=A \varepsilon(X) \times A \varepsilon(Y) \\
& =\eta(X) \times \eta(Y)=\eta\left[X \bullet_{\eta} Y\right]
\end{aligned}
$$

qed

One immediately checks that the definition extends to a smooth operation on vector fields, again denoted by $\bullet$. The relevant properties of the vector product can easily be transferred to the induced vector product:

Proposition 5.2 For all vector fields $X, Y, Z$, we have

$$
\begin{aligned}
X \bullet Y & =-Y \bullet X \\
\langle X \bullet Y, Z\rangle & =\langle X, Y \bullet Z\rangle \\
X \bullet(Y \bullet Z) & =\langle X, Z\rangle Y-\langle X, Y\rangle Z
\end{aligned}
$$

and the Jacobi identity

$$
X \bullet(Y \bullet Z)+Y \bullet(Z \bullet X)+Z \bullet(X \bullet Y)=0 .
$$

Proof Straightforward.

qed

Remark 1. The same product can be defined using the standard wedge product and the Hodge operator w.r.t. $q$. In fact, $X \bullet Y=*(X \wedge Y)$.

2. Note that, by assumption, $\Sigma$ is parallelizable, whence even globally defined frames exist. 


\subsection{Weingarten Mapping}

The second ingredient of the Ashtekar connection, the Weingarten mapping, corresponds to the second fundamental form (also called exterior curvature by physicists). It is defined by

$$
\begin{aligned}
W: T \Sigma & \longrightarrow T \Sigma . \\
X & \longmapsto{ }^{4} \nabla_{X} n
\end{aligned}
$$

Here, $n$ is the normal to $\Sigma$ within $(M, g)$, and ${ }^{4} \nabla$ is the Levi-Civita connection for $g$ on $M$. By metricity of ${ }^{4} \nabla$, the Weingarten mapping is well defined 2 Moreover it is symmetrid 3 and $C^{\infty}(\Sigma)$-linear. The second fundamental form $K$ is given by $K(X, Y)=\langle W(X), Y\rangle$. The other way round, this equation allows to obtain $W$ from $K$ as $q$ is non-degenerate.

\subsection{Ashtekar Connection}

Definition 5.2 The Ashtekar connection w.r.t. Barbero-Immirzi parameter $\beta \in \mathbb{C}$ is defined by

$$
\nabla_{X}^{A} Y:=\nabla_{X} Y+\beta W(X) \bullet Y
$$

We consider only the case $\beta \neq 0$. Note that the imaginary part of $\beta$ may be non-zero. Indeed, originally, $\beta$ has been set to i as only then some unpleasant term within the Hamiltonian constraint, prefactored by $\left(1+\beta^{2}\right)$, disappeared. Of course, if $\beta$ is not real, then, in what follows, the connections etc. will live in the corresponding complexified structures. If necessary, we will tacitly assume this (without further indication).

Proposition 5.3 $\nabla^{A}$ is metric and obeys the Leibniz rule

$$
\nabla_{X}^{A}(Y \bullet Z)=\nabla_{X}^{A} Y \bullet Z+Y \bullet \nabla_{X}^{A} Z
$$

Proof 1. The metricity of the Levi-Civita connection just reads as

$$
X\langle Y, Z\rangle=\left\langle\nabla_{X} Y, Z\right\rangle+\left\langle Y, \nabla_{X} Z\right\rangle
$$

for all vector fields $X, Y, Z$. Additionally,

$$
\begin{aligned}
0 & =\langle W(X) \bullet Y, Z\rangle+\langle Y \bullet W(X), Z\rangle \\
& =\langle W(X) \bullet Y, Z\rangle+\langle Y, W(X) \bullet Z\rangle
\end{aligned}
$$

by Proposition 5.2. Adding this to the equation above, we get

$$
X\langle Y, Z\rangle=\left\langle\nabla_{X}^{A} Y, Z\right\rangle+\left\langle Y, \nabla_{X}^{A} Z\right\rangle,
$$

hence metricity of the Ashtekar connection.

2. To prove the Leibniz rule, add the Leibniz rule

$$
\nabla_{X}(Y \bullet Z)=\nabla_{X} Y \bullet Z+Y \bullet \nabla_{X} Z
$$

for the Levi-Civita connection (see Appendix $\underline{\text { A) }}$ to

$$
W(X) \bullet(Y \bullet Z)=(W(X) \bullet Y) \bullet Z+Y \bullet(W(X) \bullet Z)
$$

which is immediately derived from Jacobi's identity and the antisymmetry of $\bullet$.

qed

\footnotetext{
${ }^{2}$ We have $W(X) \in T \Sigma$, because $\langle W(X), n\rangle \equiv\left\langle{ }^{4} \nabla_{X} n, n\right\rangle=\frac{1}{2} X\langle n, n\rangle=0$.

${ }^{3}$ See $\langle W(X), Y\rangle \equiv\left\langle{ }^{4} \nabla_{X} n, Y\right\rangle=-\left\langle n,{ }^{4} \nabla_{X} Y\right\rangle=-\left\langle n,{ }^{4} \nabla_{Y} X\right\rangle+\langle n,[Y, X]\rangle=\left\langle{ }^{4} \nabla_{Y} n, X\right\rangle \equiv\langle W(Y), X\rangle$, using metricity and torsion-freeness of the Levi-Civita connection.
} 


\subsection{Reconstruction}

It is possible to reconstruct both the metric $q$ and the second fundamental form $K$ from $E$ and $\nabla^{A}$. In fact, first reconstruct $q$ from $E$ as in Section 4 . Then, this metric $q$ determines the Levi-Civita connection $\nabla$. From this, as $\beta \neq 0$, we may regain $W(X) \bullet Y$ for all vector fields $X, Y \in T \Sigma$. Since $2 \mathfrak{x}=\sum_{i} \mathfrak{e}_{i} \times\left(\mathfrak{x} \times \mathfrak{e}_{i}\right)$ for the standard orthonormal basis $\left(\mathfrak{e}_{1}, \mathfrak{e}_{2}, \mathfrak{e}_{3}\right)$ of $\mathbb{R}^{3}$, we get

$$
W(X)=\frac{1}{2} \sum_{i=1}^{3} e\left(\mathfrak{e}_{i}\right) \bullet\left(W(X) \bullet e\left(\mathfrak{e}_{i}\right)\right) .
$$

As already mentioned, the second fundamental form can now easily be derived from this $W$.

\subsection{Curvature}

Proposition 5.4 The torsion of the Ashtekar connection is given by

$$
T^{A}(X, Y)=\beta[W(X) \bullet Y-W(Y) \bullet X]
$$

and the curvature by

$$
\begin{aligned}
R^{A}(X, Y) Z=R(X, Y) Z & +\beta\left[\left(\nabla_{X} W\right) Y-\left(\nabla_{Y} W\right) X\right] \bullet Z \\
& +\beta^{2}[W(X) \bullet W(Y)] \bullet Z
\end{aligned}
$$

Proof 1. The torsion of the Ashtekar connection is given by

$$
\begin{aligned}
T^{A}(X, Y) & =\nabla_{X}^{A} Y-\nabla_{Y}^{A} X-[X, Y] \\
& =\nabla_{X} Y-\nabla_{Y} X-[X, Y]+\beta W(X) \bullet Y-\beta W(Y) \bullet X \\
& =\beta W(X) \bullet Y-\beta W(Y) \bullet X
\end{aligned}
$$

as the Levi-Civita connection is torsion-free.

2. For the curvature observe (with $W$ rescaled by $\beta W$ )

$$
\begin{aligned}
R^{A}(X, Y) Z= & \nabla_{X}^{A} \nabla_{Y}^{A} Z-\nabla_{Y}^{A} \nabla_{X}^{A} Z-\nabla_{[X, Y]}^{A} Z \\
= & \nabla_{X} \nabla_{Y} Z-\nabla_{Y} \nabla_{X} Z-\nabla_{[X, Y]} Z \\
& +\nabla_{X}(W(Y) \bullet Z)-W(Y) \bullet \nabla_{X} Z \\
& -\nabla_{Y}(W(X) \bullet Z)+W(X) \bullet \nabla_{Y} Z \\
& -W([X, Y]) \bullet Z \\
& +W(X) \bullet(W(Y) \bullet Z)-W(Y) \bullet(W(X) \bullet Z) \\
= & \nabla_{X} \nabla_{Y} Z-\nabla_{Y} \nabla_{X} Z-\nabla_{[X, Y]} Z \\
& +\nabla_{X}(W(Y)) \bullet Z-\nabla_{Y}(W(X)) \bullet Z \\
& -W\left(\nabla_{X} Y-\nabla_{Y} X\right) \bullet Z \\
& +W(X) \bullet(W(Y) \bullet Z)+W(Y) \bullet(Z \bullet W(X)) \\
= & R(X, Y) Z+\left[\left(\nabla_{X} W\right) Y-\left(\nabla_{Y} W\right) X\right] \bullet Z \\
& +[W(X) \bullet W(Y)] \bullet Z .
\end{aligned}
$$

qed

A few of the symmetries of the Riemann curvature tensor survive the transition to Ashtekar connections. As $\nabla^{A}$ is a covariant derivative, we have

$$
R^{A}(X, Y)=-R^{A}(Y, X)
$$

and as $\nabla^{A}$ is metric, we get

$$
\left\langle R^{A}(X, Y) Z, W\right\rangle=-\left\langle R^{A}(X, Y) W, Z\right\rangle .
$$




\subsection{Frame Bundle Connection}

Taking the canonical action $\rho$ of $\operatorname{Gl}(n)$ and its subgroups on $\mathbb{R}^{n}$, we may consider the tangent bundle as an associated bundle of the frame bundle via the usual isomorphism

$$
\begin{aligned}
\mathrm{Gl}(\Sigma) \times(\mathrm{Gl}(n), \rho) & \mathbb{R}^{n} \cong T \Sigma \\
{[e, \mathfrak{x}] } & \longmapsto e(\mathfrak{x})
\end{aligned}
$$

This isomorphism induces an isomorphism between the space of covariant derivatives on $T \Sigma$ and the space of connection 1-forms in the frame bundle. Similarly, given any metric $q$ on $\Sigma$, we have

$$
\mathrm{O}_{q}(\Sigma) \times_{(\mathrm{O}(n), \rho)} \mathbb{R}^{n} \cong \mathrm{O}_{q}^{+}(\Sigma) \times_{(\mathrm{SO}(n), \rho)} \mathbb{R}^{n} \cong T \Sigma
$$

for the (oriented) orthonormal frame bundle, and an isomorphism between the metric covariant derivatives on $T \Sigma$ and connections in $\mathrm{O}_{q}(\Sigma)$. Therefore, we can now naturally transfer the Ashtekar connection from $T \Sigma$ to the frame bundles. Given a local frame $e$ on $U \subseteq \Sigma$, we may define a local connection 1-form

$$
A:=\left\langle\nabla^{A} e, e\right\rangle: T U \longrightarrow \mathfrak{g l}(3) .
$$

A bit more explicitly, for each vector field $X$, we get a $\mathfrak{g l}(3)$ matrix $A(X)$ by

$$
\langle A(X) \mathfrak{x}, \mathfrak{y}\rangle_{\text {Eucl }}=\left\langle\nabla_{X}^{A}[e(\mathfrak{x})], e(\mathfrak{y})\right\rangle
$$

for all $\mathfrak{x}, \mathfrak{y} \in \mathbb{R}^{3}$, viewing both sides as functions on $U$. The Ashtekar connection $\omega_{A}$ in the frame bundle is now the connection that is mapped to $A$ by the different pull-backs $f^{*} \omega_{A}$. Here, we viewed the frame $f$ as a mapping from some $U$ to $\operatorname{Gl}(\Sigma)$. If we assume $e$ to be $q$-orthonormal, then $\omega_{A}$ is even a connection in the orthonormal frame bundle $\mathrm{O}_{q}(\Sigma)$, and its local versions $A$ are $\mathrm{O}(3)$ connections. Indeed, if $e$ is orthonormal, then for fixed $\mathfrak{x}, \mathfrak{y} \in \mathbb{R}^{3}$

$$
\begin{array}{rlr}
\langle A(X) \mathfrak{x}, \mathfrak{y}\rangle_{\text {Eucl }} & =\left\langle\nabla_{X}^{A}[e(\mathfrak{x})], e(\mathfrak{y})\right\rangle \\
& =\nabla_{X}^{A}\langle e(\mathfrak{x}), e(\mathfrak{y})\rangle-\left\langle e(\mathfrak{x}), \nabla_{X}^{A}[e(\mathfrak{y})]\right\rangle & \\
& =\nabla_{X}^{A}\langle\mathfrak{x}, \mathfrak{y}\rangle_{\text {Eucl }}-\left\langle\nabla_{X}^{A}[e(\mathfrak{y})], e(\mathfrak{x})\right\rangle & \text { (by metricity of } \left.\nabla^{A}\right) \\
& =-\langle\mathfrak{x}, A(X) \mathfrak{y}\rangle_{\text {Eucl }} .
\end{array}
$$

Similarly, in the oriented case, we get $\mathrm{SO}(3)$ connections. From the definitions, we get

$$
\omega_{A}=\omega_{L C}+\beta \widetilde{K}
$$

with

$$
\widetilde{K}(X)=\left\langle W\left(\pi_{*} X\right), e \bullet e\right\rangle
$$

for all vector fields $X$ on the frame bundle, $\pi$ being the bundle projection.

\subsection{Physics Notation}

In the physics literature, Ashtekar connections are given by their components in some coordinate system. To reproduce this, let $\left\{M_{1}, M_{2}, M_{3}\right\}$ be a basis of $\mathfrak{s o}(3)$ with $\left[M_{i}, M_{j}\right]=\varepsilon_{i j}{ }^{k} M_{k}$, and let $\chi: U \longrightarrow \mathbb{R}^{3}$ be some chart for open $U \subseteq \Sigma$. Then we get a local basis $\left\{\partial_{1}, \partial_{2}, \partial_{3}\right\}$ for the tangent space. Finally, choose some orthonormal frame $e$, viewed as a map from $U$ to $\mathrm{O}_{q}^{+}(\Sigma)$. Hiding the dependence on $U$, we get $e\left(\mathfrak{e}_{i}\right)=: e_{i}^{a} \partial_{a}$, with $\mathfrak{e}_{i}$ being the $i$-th vector in the standard basis of $\mathbb{R}^{3}$. It is now a bit lengthy, but straightforward [12] to show that

$$
e^{*} \omega_{L C}\left(\partial_{a}\right)=\frac{1}{2} \varepsilon_{i j}^{k}\left\langle\nabla_{\partial_{a}} e\left(\mathfrak{e}_{i}\right), e\left(\mathfrak{e}_{j}\right)\right\rangle M_{k}=\Gamma_{a}^{k} M_{k}
$$

reproduces the quantity $\Gamma_{a}^{k}$ that often is called spin connection (whose geometric definition will be given in Section [6). Similarly, one checks [12] that

$$
e^{*} \widetilde{K}\left(\partial_{a}\right)=\varepsilon^{i j k}\left\langle W\left(\partial_{a}\right), e\left(\mathfrak{e}_{i}\right)\right\rangle\left[M_{j}, M_{k}\right]=k\left(\partial_{a}, \partial_{b}\right) e_{i}^{b} M^{i}=k_{a}^{i} M_{i}
$$


reproduces the extrinsic curvature $k_{a}^{i}$. Altogether, we have

$$
e^{*} \omega_{A}\left(\partial_{a}\right)=\left(\Gamma_{a}^{i}+\beta k_{a}^{i}\right) M_{i} .
$$

As an $\mathfrak{s o}(3)$ Lie algebra basis can always be regarded also as an $\mathfrak{s u}(2)$ Lie algebra basis, the expression above is also a local coordinate version of the $\mathrm{SU}(2)$ Ashtekar connection. The deeper geometric meaning of this transition is encoded in a spin structure to be considered below.

\section{Spin Structures}

Definition 6.1 A spin structure [7] on $(\Sigma, q)$ is a pair $(S(\Sigma), \Lambda)$ consisting of

- an $\mathrm{SU}(2)$ principal fibre bundle $\widetilde{\pi}: S(\Sigma) \longrightarrow \Sigma$ and

- a double covering $\Lambda: S(\Sigma) \longrightarrow \mathrm{O}_{q}^{+}(\Sigma, q)$,

such that the following diagram commutes:

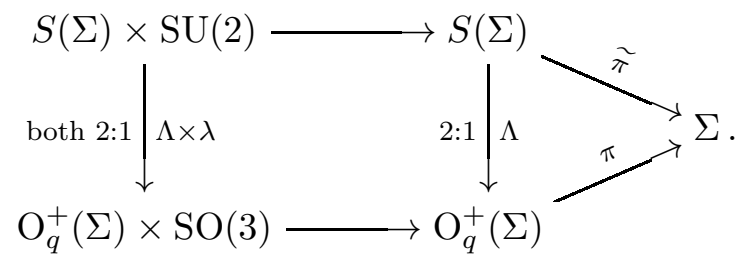

The horizontal lines denote the action of the respective structure group, and $\lambda$ the double cover $\lambda: \mathrm{SU}(2) \cong \mathrm{Spin}(3) \longrightarrow \mathrm{SO}(3)$.

As $\lambda_{*}: \mathfrak{s u}(2) \longrightarrow \mathfrak{s o}(3)$ is an isomorphism, any connection $\omega$ in $\mathrm{O}_{q}^{+}(\Sigma)$ can be lifted to a connection $\widetilde{\omega}$ via

$$
\lambda_{*} \circ \widetilde{\omega}:=\Lambda^{*} \omega .
$$

The spin bundle connection $\widetilde{\omega}_{L C}$ corresponding to the Levi-Civita connection is called spin connection, and the spin bundle connection $\widetilde{\omega}_{A}$ corresponding to the $\mathrm{SO}(3)$ Ashtekar connection $\omega_{A}$ is the celebrated $\mathrm{SU}(2)$ Ashtekar connection. Completely analogously to the case of frame bundles, the tangent bundle is associated to the spin bundle via

$$
T \Sigma \cong S(\Sigma) \times(\mathrm{SU}(2), \rho \circ \lambda) \mathbb{R}^{3} \text {. }
$$

Hence, if we assumed connections in a principal fibre bundle to be the fundamental ones, for the Ashtekar connection acting on the tangent bundle it did not matter, whether we had derived it from the $\mathrm{SU}(2)$ or the $\mathrm{SO}(3)$ version.

\section{Application to Cosmology}

Let us finally discuss highly symmetric models as used in quantum cosmology. Here, we would like to restrict ourselves to Friedmann-Robertson-Walker models. In other words, we assume that the spacetime manifold $M$ is split as $M=I \times \Sigma$, where $I$ is some open interval in $\mathbb{R}$, and the spacetime metric is given by

$$
g=-\mathrm{d} \tau^{2}+a^{2} q .
$$

Here, $q$ is a metric on $\Sigma$ of constant sectional curvature $\kappa$, and $a: I \longrightarrow \mathbb{R}$ is the scale factor. 
Observe that the normal $n$ to the Cauchy slice equals $\partial_{\tau}$. Using the Koszul formula with $[X, Y]=[Y, n]=[n, X]=0$, we get

$$
\begin{aligned}
2\langle W(X), Y\rangle & \equiv 2\left\langle{ }^{4} \nabla_{X} n, Y\right\rangle=X\langle n, Y\rangle-Y\langle n, X\rangle+n\langle X, Y\rangle \\
& =\partial_{\tau}\left[a^{2} q(X, Y)\right]=2 \dot{a} a q(X, Y)=2\left\langle\frac{\dot{a}}{a} X, Y\right\rangle,
\end{aligned}
$$

hence

$$
W=h \mathbf{1}
$$

with the Hubble "constant"

$$
h:=\frac{\dot{a}}{a}
$$

Thus, the Ashtekar connection has a very simple form

$$
\nabla_{X}^{A} Y=\nabla_{X} Y+\beta h X \bullet Y .
$$

Its torsion and curvature are given by

$$
\begin{aligned}
T^{A}(X, Y) & =2 \beta h X \bullet Y \\
R^{A}(X, Y) Z & =\left[(\beta h)^{2}-\kappa\right](X \bullet Y) \bullet Z
\end{aligned}
$$

In fact, the torsion formula follows immediately from Proposition 5.4. For the second one, observe additionally that a space of constant sectional curvature $\kappa$ fulfills [11]

$$
R(X, Y) Z=\kappa(\langle Z, Y\rangle X-\langle Z, X\rangle Y)=\kappa Z \bullet(X \bullet Y) .
$$

\section{A Leibniz Rule for Levi-Civita Connection}

In this appendix we are going to show that for all $X, Y, Z \in T \Sigma$ the Leibniz rule

$$
\nabla_{X}(Y \bullet Z)=\nabla_{X} Y \bullet Z+Y \bullet \nabla_{X} Z
$$

holds for the Levi-Civita connection. For this, let $\left(\mathfrak{e}_{1}, \mathfrak{e}_{2}, \mathfrak{e}_{3}\right)$ be the standard basis of $\mathbb{R}^{3}$ and let $e$ be some orthonormal frame. Define $e_{i}:=e\left(\mathfrak{e}_{i}\right)$ for $i=1, \ldots, 3$. Then we have to show that for all $j, k, l$ and $X$

$$
\begin{aligned}
\left\langle\nabla_{X} e_{l}, e_{j} \bullet e_{k}\right\rangle & +\left\langle\nabla_{X} e_{j}, e_{k} \bullet e_{l}\right\rangle+\left\langle\nabla_{X} e_{k}, e_{l} \bullet e_{j}\right\rangle \\
= & -\left\langle\nabla_{X}\left(e_{j} \bullet e_{k}\right), e_{l}\right\rangle+\left\langle\nabla_{X} e_{j} \bullet e_{k}, e_{l}\right\rangle+\left\langle e_{j} \bullet \nabla_{X} e_{k}, e_{l}\right\rangle=0 .
\end{aligned}
$$

In fact, first observet that

$$
\left\langle\nabla_{X} e_{i}, e_{i}\right\rangle=\nabla_{X}\left\langle e_{i}, e_{i}\right\rangle-\left\langle e_{i}, \nabla_{X} e_{i}\right\rangle=-\left\langle\nabla_{X} e_{i}, e_{i}\right\rangle
$$

for $X \in T \Sigma$, since $\nabla$ is metric, $q$ is symmetric, and $\left\langle e_{i}, e_{i}\right\rangle \equiv 1$; consequently,

$$
\left\langle\nabla_{X} e_{i}, e_{i}\right\rangle=0
$$

Then we consider the four relevant cases:

1. If $j, k, l$ are pairwise different, then the right-hand sides of the scalar products in the upper line of (1) are always plus/minus the basis element at the respective left-hand side, whence each scalar product vanishes by (2).

2. If $j=k$, then the second and the third scalar product cancel each other due to the antisymmetry of $\bullet$. For the same reason, the first scalar product in (1) vanishes.

3. If $j=l$, then the first and the second scalar product cancel; the third one vanishes.

4. If $k=l$, then the first and the third scalar product cancel; the second one vanishes.

\footnotetext{
${ }^{4}$ Note that we do not use summation convention here.
} 


\section{Acknowledgments}

The authors thank Johannes Brunnemann, Stefan Funkner, Sören Graupner and, in particular, Thomas Leistner for interesting discussions as well as Maximilian Hanusch and Matthias Schmidt for their helpful comments on a draft version of this article. One of the authors $[\mathrm{CF}]$ has been supported by the Emmy-Noether-Programm of the Deutsche Forschungsgemeinschaft under grant FL 622/1-1.

\section{References}

[1] Abhay Ashtekar: New Hamiltonian formulation of general relativity. Phys. Rev. D36 (1987) 1587-1602.

[2] Abhay Ashtekar: New Variables for classical and quantum gravity. Phys. Rev. Lett. 57 (1986) 2244-2247.

[3] Fernando Barbero: Real Ashtekar Variables for Lorentzian Signature Space-Times. Phys. Rev. D51 (1995) 5507-5510. e-print: gr-qc/9410014.

[4] Antonio N. Bernal and Miguel Sánchez: Further Results on the smoothability of Cauchy hypersurfaces and Cauchy time functions. Lett. Math. Phys. 77 (2006) 183-197. e-print: gr-qc/0512095.

[5] Antonio N. Bernal and Miguel Sánchez: On Smooth Cauchy Hypersurfaces and Geroch's Splitting Theorem. Commun. Math. Phys. 243 (2003) 461-470. e-print: gr-qc/0306108.

[6] Yvonne Fourès-Bruhat: Théorème d'existence pour certains systèmes d'équations aux dérivées partielles non linéaires. Acta Math. 88 (1952) 141-225.

[7] Thomas Friedrich: Dirac-Operatoren in der Riemannschen Geometrie. Vieweg, 1997.

[8] Robert Geroch: Domain of Dependence. J. Math. Phys. 11 (1970) 437-449.

[9] Giorgio Immirzi: Real and complex connections for canonical gravity. Class. Quant. Grav. 14 (1997) L177-L181. e-print: gr-qc/9612030.

[10] Robion C. Kirby: The Topology of 4-Manifolds (Lecture Notes in Mathematics 1374). Springer, 1989.

[11] Shoshichi Kobayashi and Katsumi Nomizu: Foundations of Differential Geometry, vol. 1. Wiley, New York, 1963.

[12] Philipp Levermann: Geometrie des Ashtekar-Zusammenhangs (Diplomarbeit). Universität Hamburg, 2009.

[13] Hans Ringström: The Cauchy Problem in General Relativity (ESI Lectures in Mathematics and Physics). European Mathematical Society, 2009.

[14] Norbert Straumann: General Relativity: With Applications to Astrophysics. Springer, 2004.

[15] Thomas Thiemann: Quantum Spin Dynamics (QSD). Class. Quant. Grav. 15 (1998) 839-873. e-print: gr-qc/9606089.

[16] Robert M. Wald: General Relativity. Chicago University Press, 1984. 